\title{
LA FILOSOFÍA DE CAFÉ: EL PRIMER CAFÉ FILOSÓFICO EN MÉXICO
}

\section{The PHILOSOPHY OF 'CAFÉ': THE FIRST PHILO CAFÉ IN MEXICO CITY}

\author{
ESTHER CHARABATI \\ eticacotidiana@gmail.com \\ Universidad Nacional Autónoma de México \\ ORCID: https://orcid.org/0000-0001-8608-7925
}

RECIBIDO: 7 DE ABRIL DE 2019

ACEPTADO: 11 DE SEPTIEMBRE DE 2019

Resumen: Conversar es una actividad libre en la que reconstruimos nuestras experiencias y las recreamos y analizamos con ayuda del interlocutor. Un "arte del instante" cuyo único fin es el placer. Desde su surgimiento en las ciudades, los cafés se convirtieron en espacios privilegiados para sociabilizar y conversar, y México no fue la excepción. Una manera de unir la conversación con la filosofía fue la creación de los cafés filosóficos. En México, desde hace dieciocho años, semanalmente se desarrolla un café fillosófico en la Cafebrería El Péndulo.

Palabras clave: café filosófico, conversación, cafés, cafés en México

\begin{abstract}
Conversation is an activity that we freely carry out. Talk allow us to reconstruct, create anew and analize our experiences with the help of one or several interlocutors. An "art of the moment" whose sole purpose is pleasure. Since its emergence in the cities, cafes became privileged spaces for conversation, dialogue and even fierce but cordial debate, places to socialize. Mexico was no exception. A way to link the conversation with the philosophy was the creation of the philosophical cafes, and in Mexico City, for eighteen years, a philosophical cafe takes place weekly at one of the locations of Cafebrería El Péndulo.
\end{abstract}

Keywords: Philocafé, conversation, cafes- cafés in Mexico 


\section{La conversación, un placer banal}

La conversación, esa experiencia lingüística tan despreciada por su asociación con lo banal, con lo cotidiano, con el placer; esa actividad tolerante que abre la puerta a las confesiones, los chismes y los chistes, es un espacio único para reconstituir nuestras vivencias; es transformadora en el sentido de que ilumina las propias experiencias al obligarnos a encontrar el lenguaje para nombrarlas -con ello, cada narrador "se acoge a sí"-, y ofrecérselas al otro, que las recibe y también puede poner palabras en ellas. ${ }^{1}$

La conversación es una expresión de libertad, pues cada uno elige a la persona con la que quiere hablar y en ese encuentro la experiencia individual -y la exposición a la mirada del otro- se vuelve significativa y brinda a cada uno claridad sobre sí mismo.

Aunque no tiene otro fin aparte del placer, escribe Zaid, "La conversación realiza (en la conversación) lo que añora o sueña (en la realidad). Produciéndose produce, construye, educa, divulga, celebra, libera."2 Espontánea o planeada, suele convocar a cierta amabilidad por parte de quienes la practican; el objetivo es distraerse y constituye, en lo efímero, la sociedad ideal que políticos, leyes e ideologías parecen incapaces de construir. Montaigne sostenía: "El más fructuoso y natural ejercicio de nuestro espíritu es a mi ver la conversación: encuentro su práctica más dulce que ninguna otra acción de nuestra vida". ${ }^{3}$

\footnotetext{
${ }^{1}$ GIANNINI, Humberto: La reflexión cotidiana, Editorial Universitaria, Santiago de Chile, 1987, pp.88-93.

2 ZAID, Gabriel: Instituciones de la conversación. Disponible on-line en www.letraslibres.com/mexico-espana/instituciones-la-conversacion (Último acceso: diciembre 23 de 2018).

3 MONTAIGNE, Michel de: Ensayos. Disponible on-line www.cervantesvirtual.com/nd/ark:/59851/bmcqz259 (Último acceso: enero 9 de 2019).
} 
Si bien es un contrapoder a otros discursos, algunos la desprecian por fugaz: es "el arte del instante". Pero su precariedad no limita su gran ambición: establecer entre las personas una relación que conserve una distancia respetuosa y una proximidad sensible, de atención y ligereza, de seriedad y desenvoltura. Es, de acuerdo con Godo, un crucero en el que confluyen ética -por ser un arte de vivir-, política -por buscar una socialización que lleve a una microsociedad armoniosa, literatura -por intentar dar una forma adecuada a nuestros pensamientos- y filosofía -por buscar apropiarse de la felicidad y la verdad.

Aunque es una actividad libre, un juego en el que se responde "en directo" y sin reflexión, conversar exige a los participantes plegarse a ciertas reglas, por lo que constituye un espacio de progreso y civilización del individuo. ${ }^{4}$ Una de estas reglas es la pertinencia, no todo puede ser dicho en cualquier momento: "Cuando tomamos la palabra en una conversación, sin duda tenemos varios objetivos más o menos conscientes: establecer un lazo social, pasar el tiempo, sentir el placer de hablar, responder a una pregunta, tratar de presentarnos en nuestra mejor cara, etc. Entre esos objetivos, sin embargo, la preocupación de decir algo pertinente tiene un lugar importante: en el mejor de los casos, despertaremos el interés de los interlocutores, en el menor, pasaremos por personas sensatas, capaces de conversar. ${ }^{5}$

La ventaja de una buena conversación es la manera de existir que introduce: es un modo de ser con otros que permite decir cosas que no se dirían en otros espacios. Además, estimulados por la conversación, los participantes no piensan igual que cuando están

${ }^{4}$ GODO, Emmanuel: Une histoire de la conversation, PUF, Paris, 2003. Disponible on-line en http://www.emmanuel-godo.com/wpcontent/uploads/2012/05/Conversation-intro1.pdf (Último acceso: marzo 20, 2019).

${ }^{5}$ DESSALLES Jean-Louis, Les origines du langage, une histoire naturelle de la parole, Hermes Sciences publications, 2000, pp. 259-260. 
solos, o sea que son llevados a pensar algo que aún no habían pensado o que no sabían que pensaban. ${ }^{6}$ Por si fuera poco, fortalece: la conversación enseña y ejercita a un tiempo mismo. Si yo converso con un alma fuerte, con un probado luchador, este me oprime los ijares, me excita a derecha a izquierda; sus ideas hacen surgir las mías: el celo, la gloria, el calor vehemente de la disputa, me empujan y realzan por cima de mí mismo; la conformidad es cualidad completamente monótona en la conversación. ${ }^{7}$

Espacios de conversación son la calle, la plaza y el café, espacios públicos en los que todos somos iguales, al menos, al conversar. ${ }^{8}$ Quizá, volviendo a Zaid, la conversación "cayó del cielo y asustó, hasta que fue volviéndose un hogar de la tribu, un espacio acogedor y esclarecedor, donde la vida era más vida." ${ }^{9}$ De ahí, afirma el autor, nacieron diferentes tipos de instituciones -la academia, el monasterio, la universidad- y de formatos -las tertulias, los lugares de trabajo, las cantinas y los cafés, entre otrosLas tertulias son, fundamentalmente, reuniones ociosas de personas que leen y critican las ideas imperantes; son espacios en los que todas las opiniones son válidas -y refutables-. Ahí nace la filosofía; aparentemente Sócrates fue el primer filósofo en exponerse públicamente en reuniones de diversas índoles, dispuesto a escuchar a cualquiera, a que se refuten sus opiniones y a cuestionar lo que otros afirman. Sin embargo, la cultura de la conversación ha ido cambiando y no es exagerado pensar que

La vida de Café ocuparía en este proceso un lugar central, lleno de voces $\mathrm{y}$ de silencios, al ser autopercepción $\mathrm{y}$ desvelamiento,

\footnotetext{
${ }^{6}$ CAHEN, Gérald: Une manière d'être à plusieurs, en La Conversation, un art de l'instant, Autrement, coll. Mutation, Paris, 1999, pp. 77-78.

${ }^{7}$ MONTAIGNE, op.cit.

${ }^{8}$ GIANNINI, op.cit.

9 ZAID, Gabriel: Instituciones de la conversación. Disponible on-line en www.letraslibres.com/mexico-espana/instituciones-la-conversacion

(Último acceso: enero 15 de 2019)
} 
transformación e interrupción de la individualidad moderna en el escenario de una sabiduría que, sin afirmarse como tal, se hace densa en medio de charlas, ruidos y rumores, entre el zumbido y a veces el rugido de una sociedad..$^{10}$

\section{El café, lugar de encuentro}

\section{Espacios de sociabilidad}

Los diversos espacios y formas de sociabilidad que construyen los seres humanos revelan su aptitud para relacionarse en grupos más o menos estables y más o menos formalizados: algunos cuentan con estatutos y reglamentos escritos, y otros constituyen una simple reunión de habitués que van adoptando ciertos rituales. Las condiciones para que pueda darse la socialización son una formalización mínima y un espacio propio (aunque no sea siempre el mismo). Otras características que comparten las asociaciones son una adhesión voluntaria, ciertas reglas y líderes. Es interesante notar que al objetivo de la reunión se añade el placer de reencontrarse con los pares, con los que ya hay cierta familiaridad, y que lo que empieza como encuentro de amigos puede terminar como asociación. ${ }^{11}$

Los espacios se construyen en los intercambios y relaciones de la vida cotidiana, hacen historia y crean memoria. "Una ciudad afirma De Certeau- respira cuando en ella existen lugares de habla, poco importa su función oficial: el café de la esquina, la plaza del mercado, la fila de espera en el correo, el puesto de periódicos, el

\footnotetext{
${ }^{10}$ MARTÍ MONTERDE, Antoni: Poética del café, un espacio de la modernidad literaria europea, Editorial Anagrama, Barcelona, 2007. p. 13.

11 AGULHON, Maurice y BODRIGUEL, Maryvonnee: Les Associations au village. Le Paradou. Citado en GUEREÑA, J. L. "Un essai empirique qui devient un projet Raisonné". Maurice Agulhon et l'histoire de la sociabilité. Stud. hist., H. ${ }^{\mathrm{a}}$ cont., 26, Ed. Universidad de Salamanca, España, 2008, pp. 157175.
} 
portal de la escuela a la hora de la salida. ${ }^{12}$ El uso de los mismos permite su transformación de espacios en lugares y su apropiación por parte de los individuos. ${ }^{13}$ Los espacios públicos se van construyendo a través de los discursos, las prácticas y las experiencias de quienes los frecuentan y legitiman, y éstos, a su vez, se van construyendo en los contactos que brindan el esparcimiento y la conversación, además de la identificación con el lugar y lo que ahí se desarrolla.

Podríamos pensar en las tertulias y los salones como precursores de los cafés. Si bien éstos tienen sus antecedentes en La Meca, lugar en el que esta bebida fue prohibida en 1511, será en el siglo XVII cuando se cree el primer café, llamado Caffée, en Venecia ${ }^{14}$. A partir del siglo XVIII muchas ciudades se irán poblando de cafés donde artistas e intelectuales, pero también la gente común, conversa, compone el mundo y hace revoluciones "de café".

Los cafés no son sólo establecimientos en los que se sirve café, sino refugios que acogen a quienes quieren sustraerse del ajetreo cotidiano, de la familia o del trabajo, interrumpir la cotidianidad de la vida. Son espacios que anulan la oposición clásica entre ocio y trabajo. En los cafés la gente lee el periódico -hoy su celular-, escribe, se reúne a trabajar o a hacer negocios, se encuentra con sus colegas, conversa, medita... "Jamás hemos ido al café a tomar café -declara Pla-, sino a realizar un acto de sociabilidad fundamental en nuestra manera de ser.". ${ }^{15}$ A diferencia de las cadenas de cafeterías

${ }^{12}$ DE CERTEAU, Michel, GIARD, Luce y MAYOL, Pierre: La invención de lo cotidiano 2. Habitar, cocinar., Universidad Iberoamericana, México, 1999, p. 242 .

${ }^{13}$ AUGÉ, MARC: Los no lugares, Gedisa, Barcelona, 2000.

14 PENDERGRAST, Mark: El café; historia de una semilla que cambió el mundo, Argentina, Javier Vergara Editor. Citado en TÉLLEZ, Lucía, Historia cultural del consumo de café en México, Tesis de Doctorado, Universidad Veracruzana, Xalapa, 2016, p. 41.

15 PLA, Josep: El café, en: Humor Honesto y vago, citado en MARTín MONTERDE, op. cit. p. 20. 
actuales -idénticas, sin personalidad-, los cafés son uno de esos espacios que están cada vez más ausentes en las grandes ciudades; "lugares animados producidos por una historia más antigua y más lenta, donde los itinerarios individuales se cruzan y se mezclan, donde se intercambian palabras y se olvida por un instante la soledad". 16

Escritores, lectores y pintores ocupan sus lugares en solitario para la creación individual o en grupo para la construcción de ideas, de posturas, de sueños. Su comodidad -y el aliciente de una buena taza de café- los ha convertido en los lugares favoritos para la discusión política, las confidencias, los debates filosóficos y la elaboración de proyectos: verdaderos puntos de referencia para el segmento que los frecuentan. Entre sus aportes innegables están la participación ciudadana y su incidencia en el desarrollo de la cultura. ${ }^{17}$ Desde fines del siglo XVIII, en Francia los cafés son reconocidos como centro de la vida social urbana y como epicentro de sus transformaciones, pero también se va consolidando la percepción del café como una institución letrada, una especie de academia improvisada y sin sede propia. Incluso Unamuno consideraba al café como la verdadera universidad popular. ${ }^{18}$

\section{Los cafés en México}

El primer café en México abrió sus puertas a fines del siglo XVIII en la esquina de Tacuba y lo que hoy es Monte de Piedad. Desde la

\footnotetext{
${ }^{16}$ AUGÉ, MARC: op.cit. p. 38.

${ }^{17}$ ALCOTA CASTILLO, Aldo: El Café, territorio urbano para la divulgación cultural, tesis de máster, Universidad Politécnica de Valencia, 2008. Disponible en:

https://riunet.upv.es/bitstream/handle/10251/14472/Trabajo\%20tesina.\%20El\%2 0caf $\% \mathrm{C} 3 \% \mathrm{~A} 9-$

$\% 20$ territorio $\% 20$ urbano $\% 20$ para $\% 201$ a $\% 20 \% 20$ divulgac.pdf? sequence $=1 \&$ isAl lowed=y (Último acceso: marzo 18, 2019)

${ }^{18}$ MARTÍ MONTERDE, Antoni. op.cit. p. 196.
} 
puerta se ofrecía a los transeúntes café "al estilo de Francia" con leche y azúcar. Los clientes -en su mayoría extranjeros- eran mal vistos por frecuentarlos y las mujeres tenían prohibida la entrada; pronto, sectores de las clases alta y media se concentraron en sus propios cafés. ${ }^{19}$ Para el siglo XIX, los cafés ya tenían una dudosa reputación, pues "los cafés eran clubes políticos, centros de conspiración, de espionajes, refugio de cesantes, vagos, empleados, jugadores, caballeros de industria, asilo de políticos, periodistas, militares, literatos, cómicos, niños de casa rica, dueños de haciendas..." $"$.

Conjugaban reunión, diversión y pleito. Algunos, como el Café Veroly, reunían a amantes del teatro. Hubo otros, como El Café Europa (hoy Café de Nadie), que alojó a los estridentistas y fue, "al mismo tiempo, escenario, medio receptor y difusor de ideas, lugar de creación de vínculos de diferente tipo." ${ }^{~}$; ; el Café París al que llegaba "todo México", desde los muralistas hasta los Contemporáneos, desde Paz y Zea hasta Bonifaz Nuño ${ }^{22}$, o el Café

${ }^{19}$ TÉLLEZ, Lucía, Pendergrast, M. El café; historia de una semilla que cambió el mundo, Argentina, Javier Vergara Editor. Citado en L. Téllez, Historia cultural del consumo de café en México, Tesis doctoral, Universidad Veracruzana, México, p.41. Disponible en: https://cdigital.uv.mx/bitstream/handle/123456789/41692/TellezRodriguezLucia. pdf? sequence=1\&isAllowed=y (Última visita: marzo 21, 2019).

${ }^{20}$ Díaz y de Ovando, Los cafés en México en el siglo XIX, ¿Cómo eran los antiguos cafés y por qué eran tan importantes en el México decimonónico? Citado en LOZADA LEÓN, Guadalupe: Espacios de encuentro político y social en el siglo XIX. Disponible on-line en www.relatosehistorias.mx/numerovigente/como-eran-los-antiguos-cafes-y-por-que-eran-tan-importantes-en-elmexico-decimononico (Último acceso: diciembre 5, 2018)

21 FRANK, Marco y GONZÁLEZ, Alexandra Pita: El Café de nadie como espacio de sociabilidad del movimiento estridentista, Estudios sobre las Culturas Contemporáneas, Vol. XXIII. Aproximaciones a la cultura desde las ciencias sociales y humanidades II, Colima, 2017, pp. 74-75.

${ }^{22}$ CAMPOS, Marco Antonio: Café París, Enciclopedia de la literatura en México, 2018. Disponible on-line en www.elem.mx/estgrp/datos/1322 
América, poblado de estudiantes. El hecho de que los intelectuales $\mathrm{y}$ artistas se reúnan en un café abre a otros públicos menos conocedores una conversación que antes era privada y alejada de la gente. Al abandonar su "pequeño mundo" entran en contacto con lo nuevo y ellos mismos se transforman. ${ }^{23}$

Así lo recuerda Paz:

El Café París fue una sociedad dentro de la sociedad. Asimismo, una geografía: cada mesa era una tertulia, cada tertulia una isla y una plaza fortificada. Las relaciones entre las islas eran, al mismo tiempo, frecuentes y arriesgadas. Siempre había algún intrépido -o algún inconsciente- que iba de una mesa a otra. Unos eran mensajeros y otros desertores. Porque había también emigraciones y escisiones. ${ }^{24}$

En una sociedad como la mexicana, cuyas clases estaban separadas por verdaderos muros de contención, los cafés -como los bares y las cantinas- favorecieron la apropiación de esos espacios públicos por una población heterogénea, representativa de gran parte de los habitantes de la ciudad, contribuyendo a agrietar las barreras de clase. Con la mezcla de clases sociales, profesiones y edades, se propició una democratización de la vida cotidiana. ${ }^{25}$

En los dos últimos siglos, en numerosos países, los cafés se han convertido en protagonistas de la vida urbana. Son espacios polifacéticos que brindan privacidad, hospitalidad, encuentros, información, vitrinas que exhiben al cliente $\mathrm{y}$, a menudo, lo dotan del aura que poseen el café o los habitués del mismo. Lejos del

${ }^{23}$ ESPINOSA, Gabriela Mariel: Lugares de encuentro: el café y el taller literario en el México de los años '20 en Anclajes VIll. 8 (diciembre 2004), pp. 101-119. Disponible en: http://www.biblioteca.unlpam.edu.ar/pubpdf/anclajes/n08a04espinosa.pdf (Último acceso: febrero 27, 2019).

${ }^{24}$ Paz, Octavio, Convergencias, citado en: GAY, Juan Pascual: Más allá y más acá del papel en blanco. El café, el bar y las tertulias en el fin de siglo mexicano, Revista Valenciana, ${ }^{\circ} 4,2009$, p. 126.

${ }^{25}$ GAY, Juan Pascual, op.cit. pp.101-130. 
hogar, de la familia, de las aulas y de las oficinas, los cafés ofrecen tanto el anonimato como el marco para la tertulia. Ahí se piensa, se habla, se discute, se conquista, se hacen amigos y enemigos y, sobre todo, se socializa. Algunas de estas conversaciones los han convertido en un espacio privilegiado para el ejercicio del pensamiento y la difusión del pensamiento filosófico. Octavio Paz marca la diferencia que establecen los espacios: "Los de Taller frecuentábamos los bares y los cafés; los de Tierra Nueva se reunían en el jardín de la Facultad de Filosofía y Letras"26. Este recuerdo plantea dos posibilidades para la reflexión filosófica: la informalidad, el autodidactismo y el diálogo, frente a la enseñanza más formal y acartonada de las aulas.

Estamos, nos parece, ante una reivindicación de un tipo de conversación - que flota entre el debate y la discusión, la plática y el diálogo, el chisme y la disputa- que forma sin imponer; un tipo de charla que no busca enseñar pero puede ilustrar, que permite la ignorancia, el error, la opinión y el intercambio. Estamos, nos parece, frente a la "filosofía de café", una variante de la conversación que en la última década del siglo $\mathrm{XX}$ será "rehabilitada" por el café filosófico.

\section{El café filosófico}

Una noticia del 10 de diciembre de 1992 en la revista francesa VSD lleva por título:

"Sócrates y Platón. Enseña filosofía en el café". La novedad que anuncia sucede en el Café des Phares: "La filosofía sale de su ghetto. Personas anónimas acuden todos los domingos para hacerla, en el café, en torno a Marc Sautet...(..) "Quitarle a la filosofía su camisa de fuerza

\footnotetext{
${ }^{26}$ PAZ, Octavio: Antevíspera: Taller (1938-1941), en GAY, Juan Pascual, op.cit. p. 125.
} 
intelo-elitista es la ambición de ese "Sócrates" parisino... Los filósofos hoy están dramáticamente alejados de los problemas concretos, de la realidad; pasan su tiempo dando conferencias sobre la filosofía en lugar de hacerla. El colmo." 27

Hay que decir que son más las notas que cuestionan el proyecto y lo acusan de impostura, de la imposibilidad de mezclar café y filosofía.

Los asistentes a este café pasan de ser un puñado de amigos a doscientos participantes. En 1995, gracias a un apoyo, Sautet logra organizar cafés filosóficos simultáneos en 25 establecimientos. Como ningún maestro de filosofía responde a la convocatoria, serán los propios adeptos del Café des Phares quienes animen los cafés, que en pocos años se extienden a diversos países. ${ }^{28} \mathrm{Su}$ propuesta constituye una práctica de la filosofía que atestigua su frescura y que no requiere de la soledad, del silencio ni de la palabra escrita.

Los cafés, como se vio más arriba, han sido tradicionalmente lugares de intercambio de palabras, donde los pensamientos pueden ser expresados con toda libertad e invitan al otro a intervenir. Constituyen un espacio de construcción y de rebeldía contra el pensamiento único; un lugar donde la palabra vehicula las opiniones y representaciones sociales y propicia debates, comentarios y toma de posición frente a asuntos de actualidad... Sin embargo, en las grandes ciudades, los cafés se han convertido en establecimientos donde las personas consumen y se ignoran.

El café filosófico viene a restituir la tradición de conversar y discutir, ahora matizada con la carga del adjetivo "filosófico". A pesar de ser un espacio con acceso limitado -en el sentido de que es necesario pagar al menos el consumo-, el café filosófico está más

27 DIAMENT, Jacques: Les Cafés de Philosophie. Une forme inédite de socialisation par la philosophie. L'Harmattan, Paris, 2001, pp.13-14.

${ }^{28}$ DIAMENT, Jacques, op.cit. pp. 1-20. 
abierto al público que los coloquios, los seminarios y las aulas de la universidad, exclusivos para los iniciados. Schopenhauer aconsejaba a quien buscara la verdad: "que se dirija a cualquier parte menos a las universidades, porque ahí su hermana, la filosofía convencional, ejerce el mando y es la que dicta los platos del menú". ${ }^{29}$ Por su parte, Kant advertía que: "No se aprende la filosofía, no se puede aprender más que a filosofar". ${ }^{30}$

Los cafés se multiplican en un momento en que la idea de que la filosofía ha perdido su vocación se va extendiendo. En un artículo de 2016, "Cuando la filosofía perdió su camino", esta vez del New York Times, dos profesores universitarios -Robert Frodeman y Adam Briggle- denuncian la institucionalización de la filosofía y su fracaso al limitarla al ambiente académico y, "purificarla": el filósofo dejó de ser un tábano para convertirse en un "experto" con muchos conocimientos y capacidad para crear y destruir argumentos. Para estos autores, la ética, la búsqueda de la sabiduría y la vocación, se perdieron en el camino. ${ }^{31}$ Lo cierto es que la idea de que la filosofía debe salir de las aulas ha ganado adeptos, como lo muestra el libro de la UNESCO La filosofía, una escuela de la libertad $^{32}$, que presenta las diversas iniciativas, formatos y espacios para hacer filosofía "fuera de los muros".

${ }^{29}$ SCHOPENHAUER, Arthur, Sobre la filosofía de universidad, Alianza, 1991, Madrid, p. 60.

${ }^{30}$ KANT, Emanuel, Crítica de la razón pura, A838/B866, Madrid, Alfaguara, 1988, pp. 650-651.

31 FRODEMAN, Robert y Adam BRIGGLE: Cuando la filosofía perdió su camino (When philosophy losts its way) en: Letras Libres, ${ }^{\circ} 208,15$ de abril 2016. Disponible on-line en www.letraslibres.com/mexico/cuando-la-filosofiaperdio-su-camino.

(Último acceso: diciembre 3, 2018).

${ }^{32}$ GOUCHA, Moufida (dir.) La filosofía, una escuela de la libertad. Enseñanza de la filosofía y aprendizaje del filosofar: la situación actual y las perspectivas para el futuro. UNESCO, México, 2011 
El café filosófico no se plantea como alternativa a la universidad: el lugar en el que se ejerce la filosofía está estrechamente relacionado con el tipo de filosofía que se profesa: científica, experta, popular, exclusiva... El café filosófico trabaja con los materiales que circulan en un café: opiniones, prejuicios y experiencias. Su emergencia y el éxito alcanzado en numerosas ciudades, obliga a plantear la pregunta por el lugar que ocupa la filosofía en la vida cotidiana, de la que ha sido separada desde hace siglos. No es que las personas hayan dejado de preguntarse, sino que la sociedad de consumo no ofrece espacios para reflexionar en compañía. Los cafés filosóficos parecen responder a un malestar que la sociedad no ha atendido. ${ }^{33}$ En un mundo poblado de centros comerciales, de mensajes individualistas y de llamados a convertirnos en seres eficientes, la filosofía vuelve a las calles para actualizar la práctica de hacer preguntas, para restaurar el debate y el diálogo cotidianos. En ese sentido, el café es un lugar privilegiado para el debate filosófico pues crea un paréntesis en el espacio y en el tiempo para que los participantes puedan ocuparse de temas que probablemente han ido postergando. La voluntad, el amor, la verdad, la justicia aparecen de pronto como cercanos. Coincidimos con De Certeau cuando afirma: "En los cafés, en las oficinas, en los edificios, los relatos insinúan espacios diferentes. Añaden a la ciudad visible las "ciudades invisibles" de las que hablaba Calvino." ${ }^{34}$ ¿Qué es lo filosófico del café filosófico?

Para enfrentar las críticas, Sautet afirmaba que el café filosófico no es ni un círculo para iniciados ni una terapia salvaje. Más bien se

33 BLANCHARD, Jean Philippe: Memoria de la Jornada-seminario "Comprendre, introduire et animer un café philo". Disponible on-line en www.pratiquesphilo.free.fr/contribu/contrib17.htm (Último acceso 25 de enero, 2019); GALZINE, Violaine: Le café-philo: lieu de formation? Memoria de ciencias de la educación, Université Montpellier 3, 2001.

${ }^{34}$ DE CERTEAU, Michel, GIARD Luce y Pierre MAYOL, op. cit. p. 144. 
trata de un espacio alternativo de discusión y reflexión en el que no se busca la verdad, sino descubrir la complejidad de las cuestiones que se plantean y que, por serias o triviales que parezcan, son sometidas a las exigencias del razonamiento filosófico. Aunque se parte de un principio de pluralidad en el que todas las ideas son escuchadas, esto no significa que todas sean igualmente válidas: la discusión va multiplicando las dudas y cada uno tiene la oportunidad de presenciar las reacciones de los demás ante sus opiniones, para reforzarlas, desecharlas o mantenerlas. El café filosófico ha sido acusado de relativista, en el sentido de que acepta todas las ideas. Sin embargo, escucharlas no significa otorgarles el mismo valor: se relativizan las ideas, las creencias y los prejuicios, y se destaca la diferencia entre éstas y las ideas que pasaron por el tamiz de la crítica. Se trata de adoptar la postura filosófica que consiste en tomar conciencia de la relatividad de las opiniones y de las exigencias de lo verdadero, para luego dudar y tratar de fundamentar mi postura, sobre cuestiones primordiales. ${ }^{35} \mathrm{El}$ objetivo no es el consenso, sino la búsqueda de la verdad, la aceptación de la diferencia y una construcción colectiva de sentido. La respuesta a la pregunta planteada acerca de lo filosófico del café depende del concepto de filosofía de cada uno. Gramsci intercede por las muchas filosofías, al afirmar que no se puede separar la filosofía "científica" de la "vulgar y popular". Para él, la mayoría de los hombres son filósofos, por lo que la tarea consiste en volver crítica una actividad ya existente en la vida cotidiana. No se pone en duda que el filósofo profesional piensa con mayor lógica y coherencia, sistematiza mejor $\mathrm{y}$, como conoce la historia del pensamiento, puede darle un sentido al desarrollo de éste y retomar los problemas en el punto en que se encuentran.) ${ }^{36}$. Las diferencias,

${ }^{35}$ TOZZI, Michel: Penser par soi-même, Initiation à la philosophie, MICHEL TOZZI, EVO Chronique Sociale, 1996.

${ }^{36}$ GRAMSCI, Antonio: Materialismo histórico y la filosofía de Benedetto Croce, Juan Pablos, México, 1975, pp. 14-42. 
son, pues cuantitativas, al igual que en otras actividades humanas: la arquitectura, el periodismo o la administración no nacen como profesiones, sino como prácticas. El café filosófico no pretende formar profesionales; es más, ni siquiera se plantea formar, sino crear un ambiente en que se pueda conversar y debatir con cierto rigor cuestiones que atañen a los seres humanos.

Las sesiones suelen iniciar con una provocación, y a lo largo de la discusión se reformula constantemente la problemática. Hay un ir $\mathrm{y}$ venir del argumento a la experiencia: a menudo es necesario aterrizar las ideas con ejemplos para que los participantes puedan apropiárselas y propongan razonamientos que expliquen y amplíen la problemática, construyan hipótesis, las analicen y ofrezcan argumentos. Cada uno aporta desde sus recursos y conocimientos; exponer sus ideas ante los demás les da la posibilidad de tomar distancia de sus experiencias personales e interpretarlas desde una mirada social. ${ }^{37}$

Algunas de las actividades presentes en la discusión filosófica del café son:

a) problematizar el tema: presentar lo que está en juego, los conceptos y las relaciones entre conceptos, las definiciones provisionales, la delimitación del campo de aplicación, las posibles tesis y sus supuestos, las cuestiones subsecuentes, los ejes de reflexión.

b) cuestionar las afirmaciones, las definiciones, incluso las preguntas. Dejarse interpelar por las preguntas de los demás.

c) conceptualizar las nociones, redefinirlas a lo largo de la discusión.

d) argumentar, fundamentar los puntos de vista.

e) analizar casos concretos para aclarar el tema, sin quedarse en ellos.

${ }^{37}$ HAWKEN, Johanna: Philosopher avec les enfants : enquête théorique et expérimentale sur une pratique de l'ouverture d'esprit. Tesis doctoral. Universidad Panthéon-Sorbonne, Paris I, 2016, pp. 356-440. 
f) reformular una intervención para construir sentido filosóficamente, articularla con las precedentes (las refuerza, las completa, las objeta)

e) referirlo a algún filósofo de manera que se entienda aún sin conocer sus ideas.

f) elaborar síntesis parciales o una recapitulación final. ${ }^{38}$

\section{El café filosófico "a la mexicana"}

En el año 2000, tras leer una noticia periodística sobre el café filosófico de Sautet, pensé en crear un café filosófico en México. Si bien se habían organizado cafés en algunas ciudades de la república, por la información que obtuve, su carácter había sido efímero. La propuesta fue bien recibida por la Cafebrería El Péndulo, en la colonia Polanco de la Ciudad de México ${ }^{39}$.

El Péndulo de Polanco, al igual que las demás sucursales, fue pensado como tienda de libros, discos, películas y artículos de diseño, construido con la intención de "superar el estante librero y convertirlo en una experiencia integral", y de ofrecer actividades culturales. Su propósito era insertarse "en la tradición del típico café austriaco; la tertulia literaria sucede en el cafecito". 40 Esta cualidad, a la que se suma la belleza que logró el proyecto arquitectónico al remodelar una vieja casona de mediados del siglo $\mathrm{XX}$, la ha llevado a ser considerada por varias publicaciones como

38 TOZZI, Michel. Le café-philo: quelle responsabilité pour la philosophie?, 2001. Disponible on-line en: www.pratiquesphilo.free.fr/contribu/contrib02.htm (Último acceso: Enero 29, 2018).

${ }^{39}$ Se inauguró en 1997.

${ }^{40}$ GUTIÉRREZ, América: La historia detrás de El Péndulo en Chilango, 17 de junio de 2013, México. Disponible on-line en: https://www.chilango.com/cultura/el-pendulo-celebra-su-aniversario/ (Último acceso: febrero 18, 2019). 
una de las mejores librerías del mundo- ${ }^{41}$ convirtió a esta "cafebrería" en un marco inmejorable para el proyecto: paredes tapizadas de libros, un ambiente cálido, música de fondo...

Se decidió que el café filosófico se realizaría en la parte de arriba, donde se unieron unas cuantas mesas con sillas alrededor. Los encuentros serían semanales, para garantizar la continuidad; la asistencia, libre, sin consumo obligatorio. El día se eligió tomando en cuenta que la Ciudad de México suele tener mucho tránsito: los lunes en la noche parecían los días en que menos gente sale de su casa; por otro lado, consideramos que en ese día de la semana era menor el número de compromisos sociales. El horario: de 20:30 a 22 horas, un poco menos de lo que dura una clase, para no correr el riesgo de que la gente -ya cansada a esa hora, se aburriera. El animador -yo- recibiría por parte de El Péndulo un libro como pago por cada sesión.

El 11 de septiembre de 2000 -fecha imborrable por el ataque a las Torres Gemelas en Estados Unidos- tuvimos nuestro primer café filosófico en el que participaron los amigos a los que había invitado... y que, cumplido el deber de amistad, no volvieron a la siguiente semana. La casualidad hizo que unos estudiantes de Guadalajara que entraron al Péndulo vieran el cartel y subieran. El café filosófico se mantuvo de casualidades durante varios meses: en ocasiones con dos asistentes, otras con cuarenta (hasta ahora no hemos podido determinar las causas que aumentan o disminuyen la asistencia).

\footnotetext{
${ }^{41}$ The guardian, en su edición internacional del 11-01-2008, la incluyó en su lista Best bookshops. Top shelves. Disponible on-line en: www.theguardian.com/books/2008/jan/11/bestukbookshops (Último acceso: febrero 7 de 2019), y la revista Expansión de México le otorgó el primer lugar entre las 18 librerías que te dejarán enamorado de su belleza (agosto 6, 2015) Disponible on-line en www.expansion.mx/lifestyle /2015/08/06/las-libreriasmas-geniales-del-mundo (Último acceso: febrero 7, 2019).
} 
La publicidad, en un principio, no era más que un cartel con una pregunta sobre el tema. El primero fue: “Existe la verdad?”. Posteriormente, y durante años, enviamos cientos de correos a conocidos y desconocidos; en 2007 se creó un grupo en $\mathrm{Yahoo}^{42}$ en el que cada semana se inscribían los nuevos asistentes: actualmente son 1147; en 2012 se creó un grupo en Facebook que hoy cuenta con 252 miembros. Los correos y posts suelen incluir algunas preguntas para reflexionar sobre el tema y un artículo de mi autoría. Es común que participantes que no han sido invitados se animen a unirse a partir del cartel o del debate grupal que presencian desde sus lugares, como lo atestigua un cafepensador ${ }^{43}$ :

Yo había entrado a la librería a buscar un libro y vi el cartelito al pie de las escaleras. Juntar la filosofía con el café, algo tan seriotamente formal con algo tan chismolero me pareció, sin embargo, más que un sacrilegio, una divertida asociación. Entonces me puse a orejear, porque allí estaban reunidos esa noche y decidí presentarme a la semana siguiente. Así me convertí en un contertulio más del café. ${ }^{44}$

El espacio es apropiado para albergar a unas quince o veinte personas, por lo que, cuando la asistencia es mayor, la gente se sienta en los escalones o incluso se queda parada. Después de algunos años, la dinámica se vio afectada por las personas que llegaban tarde o se iban antes de que terminara el café, por lo que se decidió cobrar una cuota de 70 pesos $^{45}$, de la que una parte quedaría para la librería. Aunque no toda la gente llega puntual, a partir de entonces la sesión se desarrolla de manera fluida. En un modesto intento por volver accesible el café a todos, quien así lo requiere, es "becado"46.

\footnotetext{
${ }^{42}$ Yahoo groups. Café filosófico México. Grupo público.

${ }^{43}$ Palabra con la que nos referimos a los participantes.

${ }_{44}^{44}$ Alfredo Ortega Trillo, comunicación personal, 8 de octubre de 2009.

${ }^{45}$ Actualmente es de 100 pesos.

${ }^{46}$ No se le cobra la cuota.
} 
Desde 2003 organizamos con El Péndulo un evento para celebrar el aniversario del café, abierto el público en general. Hasta el aniversario número 18 habíamos realizado 820 cafés. La única evaluación que tenemos es la de los participantes.

¿Cómo funciona el café filosófico?

Está abierto al público, a los paseantes, a los bebedores de café; constituye un hito en la práctica filosófica por constituir un espacio para pensar y discutir filosóficamente con hombres y mujeres dispuestos a detenerse un rato para reflexionar sobre temas que les interesan y que pueden ser abordados desde una perspectiva filosófica. El propósito es que cada uno de los participantes vaya aprendiendo de su propia elaboración intelectual, de escuchar las intervenciones y de la confrontación de sus ideas con las de los demás. Un comentario de un cafepensador ilustra esta idea:

Agradezco al café porque de una manera simple ha desmitificado la aureola mística que revestía a la Filosofía, a la que sólo los "iniciados" o los filósofos entendían; ahora cualquier ser humano puede opinar y hacer Filosofía, cualquier ser humano que se siente, reflexione y opine puede hacer Filosofía. El hecho de hacer pensar a las personas, es un logro filosófico. Pero si alguien busca la verdad absoluta siempre podrá recurrir a la ciencia. ${ }^{47}$

Los retos son muchos: convocar, motivar a los asistentes a elegir los temas y a dialogar, establecer un clima de respeto y cordialidad que estimule la participación, provocar y sostener un debate de calidad, dar coherencia al entramado que se va formando, promover una actitud de escucha, entender lo que cada uno - con su estilo propio- quiere comunicar, acompañarlo para que profundice en sus ideas y mantener el humor. Esto, bajo dos condiciones fundamentales: la hospitalidad y la democracia, entendida como la posibilidad de que cada uno se exprese libremente y de que todos tengan el mismo acceso a la palabra.

${ }^{47}$ Héctor Rodriguez, comunicación personal, 7 de junio de 2004. 
El café es un espacio en el que nadie detenta la verdad, no hay respuestas buenas o malas, sino un intercambio de dudas, reflexiones y críticas en el que la única condición es la pertinencia: todos los participantes tienen derecho a intervenir y a que se les respete, siempre y cuando se ciñan al debate del momento. Así lo explica una cafepensadora:

En estos 7 años, 5 meses y 30 días he disfrutado mucho mi participación en el café filosófico, me he nutrido de experiencias, comentarios, puntos de vista antagónicos y sobre todo me he refrescado con cada una de las opiniones que comparten los cafeteros sobre un tema particular, pero sobre todo, los temas tratados que pueden parecer muy banales o simples me han hecho pensar, reflexionar, me han caído varios veintes y en ocasiones han cambiado totalmente mi manera de pensar sobre un tema. ${ }^{48}$

Quizás el mayor desafío que enfrenta el animador sea sostener el interés durante el encuentro, tomando en cuenta que no hay una exposición ni un hilo conductor inalterable: el animador requiere la flexibilidad y la habilidad necesarias para pasar de un tema a otro sin extraviarse. Además, tiene que mostrar la autoridad y la tolerancia necesarias para evitar que algún asistente monopolice el micrófono y para propiciar las intervenciones, aunque nadie está obligado a intervenir.

En los debates las fuentes están como referencia, pero evitamos el "desfile de personalidades" para centrarnos en las ideas: cada uno defiende lo que piensa basándose en argumentos, sin acudir a la autoridad del "magister dixit". No es un desprecio de la tradición ni de los filósofos, sino una estrategia para que cada uno piense por sí mismo, tal como lo plantea un cafepensador:

No importan fechas, autores ni corrientes, importa el planteamiento de preguntas, la discusión de temas y las conclusiones a las que llegue

${ }^{48}$ Juan Carlos García, comunicación personal, 31 de enero de 2019. 
cada uno en su momento de reflexión intima o pública, eso es Filosofía. Claro que un poco de documentación no hace daño, para formarnos una idea o pensamiento nuestro. ${ }^{49}$

Los cafés filosóficos suelen ser abiertos, lo que significa que no se trata de un grupo constituido, sino de un grupo que se reconfigura permanentemente. La diversidad de ideas, intereses y experiencias brinda a la reunión una gran riqueza, ya que la gente nueva evita que las dinámicas se vuelvan rutinarias. Otro elemento fundamental en los encuentros es el respeto: todos tienen derecho a discrepar, pero nadie a ofender: se atacan las ideas, no a las personas. Durante un tiempo, se requiere de un esfuerzo sostenido para lograr un clima cordial en el que las agresiones son frenadas de inmediato. Poco a poco, la dinámica del grupo va dando la pauta a las intervenciones. Así lo comenta un participante:

El café filosófico ha sido una gran experiencia: lo disfruto, aprendo escucho y tengo una sensación de pertenencia que me agrada. Se desarrolla en un ambiente amigable, en donde cada uno es respetado y escuchado y aunque no se esté de acuerdo, siempre se respeta la opinión de los demás. Los temas han sido siempre interesantes, algunos muy interesantes y a veces hasta polémicos, pero siempre disfrutables. He participado por varios años y espero seguir participando por muchos más. ${ }^{50}$

\section{¿De qué depende la calidad del debate?}

De varios factores:

\section{a. El tema}

La elección del tema tiene que responder al interés de los participantes, por lo que son éstos quienes lo eligen por votación o, al menos, lo aprueban. Si bien es cierto que el mejor tema puede empobrecerse, también lo es que un tema que sólo interese al

\footnotetext{
${ }^{49}$ Francisco Pérez, comunicación personal, 5 de octubre de 2009.

${ }^{50}$ Gabriela G Gamio, comunicación personal, 31 de enero de 2019.
} 
animador está destinado al fracaso. Le corresponde a éste formularlo y darle un enfoque particular para que resulte atractivo. Por ejemplo, si el tema es la felicidad, se puede abordar desde distintos enfoques: “¿Estamos obligados a ser felices?”, “Podemos aprender a ser felices?” o "¿Ser conscientes nos impide ser felices? De esta manera, se le da una intención y una dirección que lo acerque a las inquietudes de los asistentes.

b. Las preguntas

¿Cómo involucrar a los asistentes para que de inmediato se sientan interpelados? Iniciar con una pregunta que los sorprenda abre muchas posibilidades. En realidad, la base del debate son las buenas preguntas, es decir, las preguntas que formulamos cuidadosamente antes del encuentro, que van a las raíces de las preocupaciones humanas y que no tienen una respuesta, sino muchas, que se pueden confrontar. Cuando el animador ya está convencido de que tiene la respuesta correcta, es señal de que se trata de una pregunta pobre que, más que abrir, cierra el debate. Si tomamos el tema “¿Amigos o contactos?” la pregunta inicial podría ser: "¿Quién es tu amigo?” ¿A cuáles de tus contactos llamarías 'amigo'?". Las respuestas orientarán la primera parte del debate en un intento de definir la amistad, y los participantes se encontrarán de lleno en el tema.

c. El nivel de las intervenciones

El animador no puede contar con que las participaciones darán altura al debate porque esto es imprevisible, pero es necesario que prepare el tema con cierta profundidad, que aborde el tema desde distintas perspectivas -histórica, lingüística, artística, científica, psicológica... - y que tenga un "Plan B" por si no prende el debate. Cuando se trata de un animador con experiencia, esto sucede en forma espontánea. Una posibilidad es llevar un fragmento literario o algún otro recurso que ayude a retomar el hilo.

d. La coherencia 
Si bien un debate en un café filosófico no pretende el rigor de una clase de filosofía, dado que prioriza la libertad de expresarse, también es cierto que existe el riesgo de que se pierda el sentido de la búsqueda y acabe siendo una acumulación de ideas dispersas. El animador tiene que ir tejiendo una trama con las distintas ideas a lo largo de la sesión y, si es necesario, recapitular. No se pretende llegar a conclusiones, sino a que los participantes se lleven preguntas surgidas del análisis y el intercambio.

\section{e. El lenguaje}

De todas las causas conocidas por las que la filosofía ha ahuyentado a la gente, el lenguaje nos parece la principal. Si en un café utilizamos un lenguaje filosófico con gente que no lo domina, un alto porcentaje no nos entenderá. Cuando uno domina los conceptos, se pueden transmitir con un lenguaje cotidiano; si no los domina, más vale alejarse de ese tema. El animador no tiene la obligación de conocer todos los temas, sino de elegir aquellos que se considera capaz de abordar. En este sentido, el animador es antes que nada un buen lector, un buen divulgador $\mathrm{y}$ un buen preguntador.

f. La escucha

El animador tiene que escuchar y poner el ejemplo: se trata de acoger los pensamientos e ideas de los participantes sin prejuicios, aceptando los diferentes estilos e inquietudes. A veces es necesario reformular las intervenciones; otras, pedir que las aclaren, y otras explicar que están cambiando el tema, por lo que no se abordará dicho planteamiento.

\section{g. El diálogo}

Quizás uno de los retos más difíciles en un café consiste en saber cuándo y cómo controlar a alguien que habla mucho y/o aporta poco. Nosotros hemos optado por pedir que no se cuenten anécdotas ni experiencias personales: no siempre resulta, porque el deseo es fuerte. A algunas personas les cuesta trabajo conceptualizar y recurren al relato. Además, siempre habrá 
asistentes ofendidos porque a algunos se les dejó hablar por más tiempo. Es inevitable. Hay que combinar la tolerancia con la disciplina, en el sentido de pedir que levanten la mano, esperen su turno...

\section{h. El humor}

Como el enemigo a combatir es el aburrimiento, dos elementos clave son el humor y la provocación, quizá la ironía; por supuesto, nunca a costa de otro, sino como una manera de mantener un ambiente alegre.

\section{¿Qué motiva a los participantes a volver al café filosófico?}

Quizá lo que le da vida es precisamente lo que no es, pero que de alguna manera está presente: la posibilidad de adquirir cultura, de atender cuestionamientos existenciales, de obtener respuestas o consuelo, de debatir sus ideas sociales, de relacionarse con otras personas. ${ }^{51}$ Un comentario apunta en esa dirección:

Los temas de discusión me interesan, ya sea porque amplían mis perspectivas, ya sea porque se conectan con algo que me molesta y que no he querido abordar. Me parecieron muy agradables las personas que asisten y espero reincorporarme pronto. ${ }^{52}$

Quizás el café filosófico adquiere sentido en una sociedad individualista, acelerada, que les arrebata a las personas su propia vida para depositarla, en pedazos -salud, educación, deseos, formas de relacionarse- en manos de profesionales. La disolución de los lazos, las dificultades que la ciudad impone al encuentro y las pocas ocasiones para el diálogo que genera conocimiento $\mathrm{y}$ autoconocimiento, probablemente contribuyan a que las personas

${ }^{51}$ GOUCHA, Moufida, op.cit. pp.154-160.

${ }^{52}$ Comunicación personal, 12 de septiembre de 2015 
se interesen por la conversación, por reunirse en un café y por participar en una discusión interesante, tal como lo describe un cafepensador que es, además, un gran lector. Para él, los cafés filosóficos son:

Pequeñas ágoras urbanas ubicadas en segundos pisos o en sótanos que antes se llenaban de humo; bulliciosas e informales; atentas a lo que cada quien tiene que decir; ojos bien abiertos, oídos bien dispuestos, risas festivas, ironías, raciocinios a veces no muy bien articulados; raudos repasos de la Historia de la Filosofía; citas de los filósofos más famosos: Platón, Aristóteles. Spinoza, Kant, Nietzsche, Heidegger...Se tocan toda clase de temas, sobre todo aquellos relacionados con la Ética: Cómo debemos vivir, qué debemos hacer, qué es el Mal, por qué actuamos como actuamos.

Al final todos han aprendido algo, de manera no convencional, no académica, en plena libertad y alegría. Se han despojado de algunos de sus prejuicios, se les han abierto algunos horizontes y, desde luego, se han dotado de algunas herramientas que les permitan vivir con mayor dignidad, con mayor autenticidad y ligereza. ${ }^{53}$

\section{Filosofía en la ciudad}

Son pocas las iniciativas para organizar cafés filosóficos en la Ciudad de México y, en su mayoría, no han encontrado las condiciones para dar continuidad al proyecto o, en otros casos, para mantener la regularidad de las sesiones; tampoco existen espacios fuera de la academia -si los hay, son mínimos- en los que el Estado o la comunidad filosófica promuevan debates. ${ }^{54}$ Ante esta esta situación, en 2016 creamos el Seminario de Filosofía en la

\footnotetext{
${ }^{53}$ Isaac Abenshushan, comunicación personal, 2 de febrero de 2019.

${ }^{54}$ En 2009 se creó el Centro Educativo para la Creación Autónoma en Prácticas Filosóficas (CECAPFI), una institución particular que ofrece cursos y diplomados en este campo.
} 
Ciudad $^{55}$ en la Facultad de Filosofía y Letras de la Universidad Nacional Autónoma de México. En este seminario participan estudiantes y egresados de las carreras de Pedagogía y Filosofía que quieren llevar a la filosofía a las calles, a las plazas, buscando incidir en sectores ajenos al estudio de la filosofía a través de cafés filosóficos, talleres filosóficos e intervenciones de filosofía con niños. Esto, con miras a la ampliación de debates en sectores públicos y privados (instituciones de cultura, reclusión $\mathrm{y}$ rehabilitación, así como de asistencia infantil y de adultos mayores) y a la ampliación del campo de trabajo para los egresados de estas disciplinas.

La formación de los participantes-animadores parte del trabajo colectivo: cada uno prepara un café y lo presenta en el grupo para que éste haga críticas y sugerencias, tanto en las estrategias de animación como en las preguntas detonadoras, cómo hacer más atractivo el tema, desde qué otras perspectivas se puede abordar, si conviene o no delimitarlo... El seminario es, en primer lugar, un laboratorio, un espacio protegido -en el sentido de que las condiciones ambientales están controladas- en el que los participantes realizan investigaciones, experimentos y prácticas que luego aplicarán al animar un café filosófico externo. Asimismo, adquieren competencias para el cuestionamiento, la conceptualización y la argumentación, además de desarrollar estrategias de motivación.

En estas sesiones de preparación "Se genera una inquietud por las diferentes aristas que tiene un tema que parece común, y cómo el escuchar a los demás nos ayuda de manera positiva en la prudencia" ${ }^{, 56}$. Los participantes practican y valoran la escucha y el respeto como condición de posibilidad del conocimiento, del diálogo y de la convivencia.

\footnotetext{
55 PIFFYL 2016009 Filosofía en la Ciudad. Este apartado incorpora los comentarios de los participantes.

${ }^{56}$ Comunicación personal, Eliezer Quintas.
} 
Por otro lado, se ha constituido un equipo que trabaja como tal en la organización de eventos, preparación de cafés, ubicación de espacios, trámites, etcétera. Hasta ahora, el equipo de Filosofía en la Ciudad ha organizado cafés en librerías, cafeterías, universidades, espacios públicos e instituciones privadas. ${ }^{57}$ En 2017 instituyó el Encuentro anual de Filosofía en la ciudad ${ }^{58}$ en la Facultad de Filosofía y Letras de la UNAM y en 2018 celebró el Día Mundial de la Filosofía en la Biblioteca Vasconcelos de la Ciudad de México con un maratón filosófico. El seminario crece y sigue buscando espacios para promover el debate y la reflexión filosófica en las calles de la ciudad y, si es posible, del país.

\section{¿Qué sigue?}

Las ciudades se llenan de Starbucks y cadenas comerciales que promueven el anonimato y el individualismo, mientras los pequeños cafés, los encuentros y las conversaciones dan la pelea para sobrevivir. Los cafés filosóficos se suman a la lucha por crear espacios que inviten a la reflexión y al debate, que sean un pretexto para que las personas piensen su propia vida a partir de los grandes cuestionamientos filosóficos aplicados a los graves problemas de la actualidad, espacios donde la vida propia y la vida en común sean analizados desde lo cotidiano para pensarlos en términos más abstractos y más precisos que, de pronto, se vuelven comunes y familiares. Han pasado veintisiete años desde que surgió el primer café filosófico, en París; hace dos décadas que en México funcionan cafés filosóficos con distinta frecuencia, y ya son dos

57 Restaurante Quetzal (Oaxaca) Cooperativa Cafeína, Cafetería Encanto, Librería Salgari, Universidad de la Ciudad de México (Plantel Tezonco), Fundación Apta, Biblioteca Vasconcelos, plazas y parques de la Delegación Cuauhtémoc...

${ }^{58}$ En 2017 el tema fue "Sophía, la callejera” y en 2018 "De pecados, tentaciones y otras perversiones". 
años los que el grupo de Filosofía en la ciudad -y probablemente otros grupos- animan cafés filosóficos en cualquier espacio disponible. Confiamos en que éstos se multiplicarán dentro y fuera de las cafeterías, dentro y fuera de instituciones de todo tipo.

\section{Referencias bibliogáficas}

ALCOTA CASTILLO, Aldo: El Café, territorio urbano para la divulgación cultural, tesis de máster, Universidad Politécnica de Valencia, 2008.

BLANCHARD, Jean Philippe: Memoria de la Jornada-seminario "Comprendre, introduie et animer un café philo". Disponible online en www.pratiquesphilo.free.fr/contribu/contrib17.htm (Último acceso 25 de enero de 2019).

CAHEN, Gérald: Une manière d'être à plusieurs, en La Conversation, un art de l'instant, Autrement, coll. Mutation, Paris, 1999.

DIAMENT, Jacques: Les Cafés de Philosophie. Une forme inédite de socialisation par la philosophie. L'Harmattan, Paris, 2001.

ESPINOSA, Gabriela Mariel: "Lugares de encuentro: el café y el taller literario en el México de los años '20" en Anclajes VIll. 8 (diciembre 2004).

GALZINE, Violaine: Le café-philo: lieu de formation?, Memoria de ciencias de la educación, Université Montpellier 3, 2001.

GODO, Emmanuel: Une histoire de la conversation, PUF, Paris, 2003. Disponible on-line en http://www.emmanuel-godo.com/wpcontent/uploads/2012/05/Conversation-intro1.pdf (Último acceso: febrero 18, 2019).

FRANK, Marco y GONZÁLEZ, Alexandra Pita: "El Café de nadie como espacio de sociabilidad del movimiento estridentista", Estudios sobre las Culturas Contemporáneas, Vol. XXIII. Aproximaciones a la cultura desde las ciencias sociales y 
humanidades II, Colima, 2017. Disponible en: https://www.redalyc.org/jatsRepo

/316/31653554007/html/index.html Último acceso: marzo 18, 2019.

GUEREÑA, Jean Louis: Un essai empirique qui devient un projet Raisonné. Maurice Agulhon et l'histoire de la sociabilité Stud.

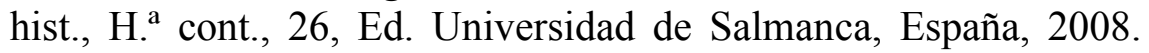
Disponible en: https://gredos.usal.es/jspui/bitstream/10366/100453/1/\%C2\%ABUn a_prueba_empirica_que_se_convierte_en.pdf (Último acceso: marzo 15, 2019).

LOZADA LEÓN, Guadalupe: "Espacios de encuentro político y social en el siglo XIX". Disponible on-line en www.relatosehistorias.mx/numero-vigente/como-eran-los-antiguoscafes-y-por-que-eran-tan-importantes-en-el-mexico-decimononico (Último acceso: diciembre 5, 2018).

MARTÍ MONTERDE, Antoni: Poética del café, un espacio de la modernidad literaria europea. Barcelona: Editorial Anagrama, 2007.

TOZZI, Michel: "Le café-philo: quelle responsabilité pour la philosophie?", 2001 Disponible on-line en:

www.pratiquesphilo.free.fr/contribu/contrib02.htm (Último acceso: Enero 29 de 2018).

ZAID, Gabriel: "Instituciones de la conversación". Disponible online en www.letraslibres.com/mexico-espana/instituciones-laconversacion (Último acceso, Febrero 15, 20019). 
